\title{
8. Über die Äquivalenz der Cesàroschen und der Hölderschen Mittel für Integrale bei negativer Ordnung.
}

\author{
Von Yoshikatsu Watanabe.
}

(Eingegangen am 2. November, 1931.)

Schon Jacob $\left({ }^{1}\right)$ hat den Äquivalenzsatz bei reeller positiver Ordnung bewiesen. Falls nun $s(x)$ in jedem endlichen Intervalle : $0 \leqq x \leqq y<+\infty$, $p$-mal stetig differenzierbar ist, so werden die genannten Mittel bei Ordnung $k(=-p+\alpha$, wo $p$ positiv ganz und $0<\alpha \leqq 1$ ist) natürlicher Weise erklärt durch

bezw.

$$
C_{k}(x)=\Gamma(k+1) \cdot \bar{C}_{k}(x)=\frac{\Gamma(k+1)}{x^{k}} \frac{d^{p}}{d x^{p}} \int_{0}^{x} \frac{(x-u)^{\alpha-1}}{\Gamma(\alpha)} s(u) d u
$$

wobei

$$
H^{k}(x)=\frac{1}{x} \int_{0}^{x} \frac{(\log x-\log u)^{\alpha-1}}{\Gamma(\alpha)} s_{p}(u) d u
$$

$$
s_{p}(x)=\sum_{n=0}^{p} \sum_{n=0}^{n}(-1)^{m}\left(\begin{array}{c}
n \\
m
\end{array}\right)(n-m+1)^{p} \frac{x^{n}}{\underline{\underline{n}}} s^{(n)}(x)
$$

ist. Aus $H^{k}(x) \rightarrow g$ für $x \rightarrow+\infty$ folgt wohl, dass $\bar{C}_{k}(x) \rightarrow \frac{g}{\Gamma(k+1)}$ im allgemeinen ist, und sogar umgekehrt für $0>k>-1$. Für $k \leqq-1(p \geqq 2)$ gilt auch die Umkehrung unter den Zusatzbedingungen, dass $s^{(l)}(x)(l=0$, $1,2, \cdots, p-2)$ beschränkt sind und noch, dass $s(x) \rightarrow g$ für $\alpha=1$ ist.

\section{$\S 1$.}

Das Cesàrosche Mittel für Integrale der Ordnung $k(>0)$ ist bekanntlich definiert durch

wobei

$$
C_{k}(x)=\frac{\Gamma(k+1) \sigma^{k}(x)}{x^{k}}
$$

$$
\sigma^{k}(x)=\int_{0}^{x} \frac{(x-u)^{k-1}}{\Gamma(k)} s(u) d u=I^{k} s(x)
$$

a.h. das Riemann-Liouvillesche verallgemeinerte Integral der Ordnung

(1) M. Jacob, Ůber die Äquivalenz der Cesàroschen und der Hölderschen Mittel für Integrale bei gleicher reeller Ordnung $k>0$, Math. Zeitschr., 26 (1927), S. 672-682. 
$k$ ist. Es ist deshalb das Integral für $k=-p+\alpha$, wo $p$ positiv ganz und $0<\alpha \leqq 1$ ist, natürlich $z u$ erklären durch

$$
\sigma^{k}(x)=I^{-p+\alpha} s(x)=D^{p} I^{\alpha} s(x)=D^{p} \int_{0}^{x} \frac{(x-u)^{\alpha-1}}{\Gamma(\alpha)} s(u) d u
$$

wenn $s(x) p$-mal stetig differenzierbar vorausgesetzt wird. Da doch, für negatives ganzes $k, C_{k}(x)$ nicht existiert, lassen wir mit Hausdorff $\left({ }^{2}\right)$ aus $C_{k}(x)$ den Faktor $\Gamma(k+1)$ fort, und setzen

$$
\bar{C}_{k}(x)=\frac{\sigma^{k}(x)}{x^{k}}=x^{p-\alpha} D^{p} \int_{0}^{x} \frac{(x-u)^{\alpha-1}}{\Gamma(\alpha)} s(u) d u,
$$

so dass an Stelle von $C_{k}(x) \rightarrow g$ die Konvergenzbeziehung

$$
\bar{C}_{k}(x) \rightarrow \frac{q}{\Gamma(k+1)}
$$

tritt. Also wenn $k=-p+1(\alpha=1)$ ist, so wird

$$
\bar{C}_{-p+1}(x)=x^{p-1} D^{p} \int_{0}^{x} s(u) d u=x^{p-1} s^{(p-1)}(x)
$$

und wenn $p=\alpha=1, k=0$ ist, so wird

$$
\bar{C}_{0}(x)=x^{0} D \int_{0}^{x} s(u) d u=s(x) .
$$

Durch teilweise Integration erhält man leicht

$$
\begin{aligned}
\sigma^{\gamma}(x)=D_{x}^{p} \int_{0}^{x} \frac{(x-u)^{\alpha-1}}{\Gamma(\alpha)} s(u) d u & =D_{x}^{p} \sum_{i=0}^{n-1} \frac{x^{\alpha+i}}{\Gamma(\alpha+i+1)} s^{(i)}(0) \\
& +D_{x}^{p} \int_{0}^{x} \frac{(x-u)^{\alpha+p-1}}{\Gamma(\alpha+p)} s^{(p)}(u) d u .
\end{aligned}
$$

Bezüglich der Limitierbarkeit von $\bar{C}_{k}(x)$ kann man voraussetzen, dass $s^{(i)}(0)=0(i=0,1,2, \cdots, p-1)$ sind, ohne Beschränkung der Allgemeinheit. Um dies zu zeigen, betrachte ich die einfachste Funktion $\varphi(x)$ $=x^{q} e^{-x}$, wo $q$ eine positive ganze Zahl oder $\mathrm{Null}$ bedeutet, für welche gilt:

$$
\varphi^{(i)}(x)=e^{-x}\left\{(-1)^{i} x^{q}+\cdots+q(q-1) \cdots(q-i+1) x^{q-i}\right\}=e^{-x} P_{i}(x),
$$

wo $P_{i}(x)$ ein Polynom $q$-ter Ordnung wird. Für diese Funktion bekommt man

$$
\bar{C}_{k}(x)=x^{p-\alpha} \sigma^{k}(x)=\sum_{i=0}^{p-1} \frac{x^{i}}{\Gamma(\alpha-p+i+1)} \varphi^{(i)}(0)+x^{p-\alpha} D^{p} I^{p+\alpha} \varphi^{(p)}(x)=v .
$$

( $\left.{ }^{2}\right)$ F. Hausdorff, Die Åquivalenz der Hölderschen und Cesàroschen Grenzwerte negativer Ordnung, Math. Zeitschr., 31 (1929), 186-196. 
Es ist aber

$$
\begin{aligned}
x^{p-\alpha} D^{p} I^{p+\alpha} \varphi^{(p)}(x) & =x^{p-\alpha} I^{\alpha} \varphi^{(p)}(x)=x^{p-\alpha} \int_{0}^{x} \frac{(x-u)^{\alpha-1}}{\Gamma(\alpha)} \varphi^{(p)}(u) d u \\
& =\int_{0}^{\sqrt{x}}+\int_{\gamma \bar{x}}^{x}=v_{1}+v_{2} .
\end{aligned}
$$

Erstens ist

$$
\begin{aligned}
& v_{1}=x^{p-\alpha}\left[\frac{(x-u)^{\alpha-1}}{\Gamma(\alpha)} \varphi^{(2-1)}(u)\right]_{0}^{\sqrt{x}}+x^{p-\alpha} \int_{0}^{\sqrt{\bar{x}}} \frac{(x-u)^{\alpha-2}}{\Gamma(\alpha-1)} \varphi^{(p-1)}(u) d u=\cdots \\
& =-\sum_{i=0}^{p-1} \frac{x^{i}}{\Gamma(\alpha-p+i+1)} \varphi^{(i)}(0)+\sum_{i=0}^{p-1} \frac{x^{i}(1-1 / \sqrt{x})^{\alpha-p+i}}{\Gamma(\alpha-p+i+1)} \varphi^{(i)}(\sqrt{x}) \\
& +x^{p-\alpha} \int_{0}^{\sqrt{x}} \frac{(x-u)^{\alpha-p-1}}{\Gamma(\alpha-p)} \varphi(u) d u
\end{aligned}
$$

wobei die erste Summation mit jener im vorigem $v$ sich aufhebt, und die zweite gegen 0 strebt für $x \rightarrow+\infty$, da $x^{i} \varphi^{(i)}(\sqrt{x})=e^{-\sqrt{x}} x^{i} P_{i}(\sqrt{x}) \rightarrow 0$ ist, während das letztere Integral, unter Anwendung des ersten Mittelwertsatzes der Integralrechnung, auf

$$
x^{p-\alpha} \frac{(x-\xi)^{\alpha-p-1}}{\Gamma(\alpha-p)} \int_{0}^{\sqrt{x}} u^{q} e^{-u} d u \quad(0<\xi<\sqrt{x})
$$

zurückgeführt wird, und daher nach dem absoluten Betrage ist

$$
\leqq \frac{1}{x-\xi}\left(1-\frac{\xi}{x}\right)^{\alpha-p}\left|\frac{1}{\Gamma(\alpha-p)}\right| \int_{0}^{\infty} u^{q} e^{-u} d u \cong \frac{1}{x}\left|\frac{1}{\Gamma(\alpha-p)}\right| \Gamma(q+1) \rightarrow 0(1)
$$

Zweitens:

$$
\begin{aligned}
v_{2} & =x^{p-\alpha} \int_{\sqrt{x}}^{x} \frac{(x-u)^{\alpha-1}}{\Gamma(\alpha)} e^{-u} P_{p}(u) d u=x^{p-\alpha} e^{-\eta} P_{p}(\eta) \frac{(x-\sqrt{x})^{\alpha}}{\Gamma(\alpha+1)} \quad(\sqrt{x}<\eta<x) \\
& =e^{-\eta} P_{p}(\eta) x^{p}\left(1-\frac{1}{\sqrt{x}}\right)^{\alpha} \cdot \frac{1}{\Gamma(\alpha+1)} \rightarrow 0(1) .
\end{aligned}
$$

Folglich ist die Funktion $x^{q} e^{-x}$ gewiss $\bar{C}_{k}$-limitierbar mit der summe 0 . Ebenso gilt auch für die Funktion

$$
\Phi(x) \equiv e^{-x} P(x) \equiv \sum_{q=0}^{p-1} \frac{a_{q}}{\underline{q}} x^{q} e^{-x} \rightarrow 0 \quad\left(\bar{C}_{k}\right) .
$$

Nun ist:

$$
\begin{aligned}
\Phi^{(i)}(0) & =D^{i}\left[e^{-x} P(x)\right]_{x=0} \\
& =(-1)^{i}\left\{P(0)-\left(\begin{array}{c}
i \\
1
\end{array}\right) P^{\prime}(0)+\left(\begin{array}{l}
i \\
2
\end{array}\right) P^{\prime \prime}(0)-\cdots+(-1)^{i} P^{(i)}(0)\right\}
\end{aligned}
$$




$$
=(-1)^{i}\left\{a_{0}-\left(\begin{array}{l}
i \\
1
\end{array}\right) a_{1}+\left(\begin{array}{l}
i \\
2
\end{array}\right) a_{2}-\cdots+(-1)^{i} a_{i}\right\} .
$$

Wenn für die gegebene Funktion $s\left(u_{i}\right), s^{(i)}(0)=c_{i} \neq 0$ für alle $i=0,1,2$, $\cdots, p-1$ oder wenigstens einige von ihnen ist, so kann man setzen:

$$
a_{0}-\left(\begin{array}{c}
i \\
1
\end{array}\right) a_{1}+\left(\begin{array}{c}
i \\
2
\end{array}\right) a_{2}-\cdots+(-1)^{i} \alpha_{i}=(-1)^{i} c_{i} \quad(i=0,1,2, \cdots, p-1)
$$

und dadurch die unbekannten Konstanten $a_{i}$ bestimmen

$$
\begin{aligned}
& a_{0}=c_{0}, a_{1}=c_{0}+c_{1}, a_{2}=c_{0}+2 c_{1}+c_{2}, \cdots, \\
& a_{i}=c_{0}+\left(\begin{array}{l}
i \\
1
\end{array}\right) c_{1}+\left(\begin{array}{l}
i \\
2
\end{array}\right) c_{2}+\cdots \cdots+c_{i}, \cdots
\end{aligned}
$$

Dann bestehen wohl die Beziehungen

$$
\Phi^{(i)}(0)=s^{(i)}(0) \quad(i=0,1,2, \cdots, p-1) .
$$

Wenn man also setzt

$$
S(u)=s(u)-\Phi(u)
$$

so wird $S^{(i)}(0)=0$ für $i=0,1.2, \cdots, p-1$, und $S(u)$ ist in Bezug auf die $C_{k}$-Limitierbarkeit ganz dasselbe wie $s(u), w . z . b . w$.

Deshalb setze ich in folgendem der Einfachheit wegen $s^{(i)}(0)=0$ $(i=0,1,2, \cdots, p-1)$ voraus. Dann wird der Ausdruck für $\sigma^{k}(x)$ bloss

$$
\sigma^{k}(x)=D^{p} I^{p+\alpha} s^{(p)}(x)=I^{\alpha} s^{(p)}(x)=\int_{0}^{x} \frac{(x-u)^{\alpha-1}}{\Gamma(\alpha)} s^{(p)}(u) d u .
$$

Wir erwähnen nun die mögliche Exweiterung der bekannten Sätze:

Lemma 1. Es sei $r>0$ und $k<0$, dann gilt

$$
\sigma^{r} \sigma^{k}(x)=\sigma^{r+k}(x), \quad \sigma^{k} \sigma^{r}(x)=\sigma^{r+k}(x) .
$$

Die erste Formel folgt aus dem bekannten Resultat $\left({ }^{3}\right)$ für $k>0$ unter Benutzung unseres Ausdrucks von $\sigma^{k}(x)$, während die zweite unmittelbar aus der bekannten Zusammensetzung der verallgemeinerten Integrale folgt.

Lemma $\mathscr{Z}$. (Konsistenssatz). Ist $\lim _{x \rightarrow \infty} C_{k}(x) \rightarrow g$ vorhanden, so ist anch $\lim _{x \rightarrow \infty} C_{k+r}(x) \rightarrow g$, wofern $k>-1, r>0$ ist.

Dies folgt zwar aus dem bekannten Satz von Chapman $\left({ }^{4}\right)$ unter Benutzung unseres Lemmas 1, wie ja schon von ihm bewiesen worden

${ }^{3}{ }^{3}$ Z. B. Hardy-Riesz, The general theory of Dirichlet's series, Cambridge Tracts, No. 18, p. 27 , Lemma 6 .

(4) S. Chapman, On non-integral orders of summability of series and integrals, Proc. Lond. Math. Soc., ser. 2, vol. 9, 1910, p. 407. Hardy-Riesz, loc. cit. p. 27, Lemma 5. 
war. Jedoch ist es nicht immer gültig, falls $k \leqq-1$ ist, wie späterhin gezeigt wird.

\section{$\S 2$.}

Das Höldersche Mittel für Integrale der Ordnung $k>0$ ist definiert durch die Formel $\left({ }^{5}\right)$ :

$$
H^{k}(x)=\frac{1}{x} \int_{0}^{x} \frac{(\log x-\log u)^{k-1}}{\Gamma(k)} s(u) d u,
$$

welche sich verwandelt, falls $x=e^{y}, u=e^{v}$ gesetzt wird, in:

$$
H^{k}(x)=e^{-y} \int_{-\infty}^{y} \frac{(y-v)^{k-1}}{\Gamma(k)} e^{v} s\left(e^{v}\right) d v=e^{-y} T_{-\infty}^{k}\left(e^{v} s\left(e^{v}\right)\right) .
$$

Die letztere Form erlaubt den negativen Wert $k=-p+\alpha$, und zwar:

$$
\begin{gathered}
H^{-p+\alpha}(x)=e^{-y} D_{y}^{p} \int_{-\infty}^{y} \frac{(y-v)^{\alpha-1}}{\Gamma(\alpha)} e^{v} s\left(e^{v}\right) d v \\
=e^{-y} D_{y}^{p}\left[\sum_{i=0}^{p-1} \frac{-(y-v)^{\alpha+i}}{\Gamma(\alpha+i+1)} \frac{d^{i}}{d v^{i}}\left(e^{v} s\left(e^{v}\right)\right)\right]_{-\infty}^{y} \\
+e^{-y} D_{y}^{p} \int_{-\infty}^{y} \frac{(y-v)^{p+\alpha-1}}{\Gamma(p+\alpha)} \frac{d^{p}}{d v^{p}}\left(e^{v} s\left(e^{v}\right)\right) d v .
\end{gathered}
$$

Falls nun $\xi^{(j)}(+0)(j=0,1,2, \cdots, p-1)$ nur beschränkt sind, so ergeben sich

$$
\begin{array}{r}
\lim _{v \rightarrow-\infty}(-v)^{\alpha+i} e^{v} \frac{d^{j} s\left(e^{v}\right)}{d\left(e^{v}\right)^{j}}=\lim _{u \rightarrow+0} u\left(\log \frac{1}{u}\right)^{\alpha+i} s^{(j)}(u)=0 \\
(i, j=0,1,2, \cdots, p-1),
\end{array}
$$

und der integrierte Teil verschwindet. Also man erhält

$$
\begin{aligned}
H^{k}(x) & =e^{-y} D_{y}^{p} I_{-\infty}^{p+\alpha}\left(D_{v}^{p} e^{v} s\left(e^{v}\right)\right)=e^{-\eta} I_{-\infty}^{\alpha}\left(D_{v}^{p} e^{v} s\left(e^{v}\right)\right. \\
& =\frac{1}{x} \int_{0}^{x} \frac{(\log x-\log u)^{\alpha-1}}{\Gamma(\alpha)}\left[\left(u \frac{d}{d u}\right)^{p}(u s(u))\right] \frac{d u}{u} .
\end{aligned}
$$

Es ist aber

$$
\left(x \frac{d}{d x}\right)^{p} f(x)=\sum_{n=1}^{p} a_{i n}^{(p)} x^{n} D^{n} f(x)\left(D=\frac{d}{d x}\right)
$$

wo

$$
a_{j i}^{(p)}=\frac{1}{n-1}\left\{n^{p-1}-\left(\begin{array}{c}
n-1 \\
1
\end{array}\right)(n-1)^{p-1}+\left(\begin{array}{c}
n-1 \\
2
\end{array}\right)(n-2)^{p-1}-\cdots \cdot+(-1)^{n-1}\right\}
$$

(5) M. Jacob, loc. cit. 
ist, wie man leicht durch vollständige Induktion ableiten kann. Also für unseren Fall ergibt sich

$$
(x D)^{n}(x s(x))=\sum_{n=1}^{p} \alpha_{n}^{(n)} x^{n}\left\{x s^{(n)}(x)+n s^{(n-1)}(x)\right\}=x \sum_{n=0}^{p} b_{n}^{(p)} x^{n} s^{(n)}(x),
$$

wobei

$$
\begin{aligned}
& b_{n}^{(p)}=\alpha_{n}^{(p)}+(n+1) \alpha_{n+1}^{\langle p\rangle}=\frac{1}{n-1} \sum_{m=0}^{n-1}(-1)^{m}\left(\begin{array}{c}
n-1 \\
m
\end{array}\right)(n-m)^{m-1} \\
& +\frac{n+1}{n} \sum_{m=0}^{n}(-1)^{m}\left(\begin{array}{c}
n \\
m
\end{array}\right)(n-m+1)^{p-1} \\
& =\frac{1}{n} \sum_{m=0}^{n}(-1)^{m}\left(\begin{array}{c}
n \\
m
\end{array}\right)(n-m+1)^{p}=\alpha_{n+1}^{(p+1)} \quad\left(b_{0}^{(p)}=b_{p}^{(p)}=1\right)
\end{aligned}
$$

ist. Gesetzt folglich

$$
\sum_{i=0}^{p} b_{i n}^{(p)} x^{n} g^{(n)}(x)=s_{p}(x)
$$

so nimmt das Höldersche Mittel der Ordnung $k(=-p+\alpha)$ die übersichtliche Form

$$
H^{k}(x)=\frac{1}{x} \int_{0}^{x} \frac{(\log x-\log u)^{\alpha-1}}{\Gamma(\alpha)} s_{p}(u) d u
$$

an. Insbesondere gilt für $p=\alpha=1$

$$
H^{0}(x)=\frac{1}{x} \int_{0}^{x}\left\{s(u)+u s^{\prime}(u)\right\} d u=s(x)
$$

Die vorstehende Definition braucht keineswegs die Voraussetzung $s^{(j)}(0)=0$. Jedoch kann dies wieder vorausgesetzt werden, obne dadurch Veränderung der $H^{*}$-Limitierbarkeitseigenschaft hervorzurufen. Wenn. nämlich $\varphi(x)=x^{q} e^{-x}$ ist, wo $q$ positiv ganz oder Null ist, so wird $\varphi^{(n)}(x)$ $=e^{-x} P_{n}(x)$, wo $P_{n}(x)$ ein Polynom der Ordnung $q$ bedentet. Für diese Funktion gilt

$$
\varphi_{p}(x)=\sum_{n=0}^{p} b_{n}^{(p)} x^{n} \varphi^{(n)}(x)=\sum_{n=0}^{p} \sum_{m=0}^{q} c_{n m} x^{m+n} e^{-x} \rightarrow 0
$$

für $x \rightarrow \infty$. Infolgedessen soll ihr Höldersches Mittel der Ordnung $a>0$ auch gegen 0 streben, also

$$
H^{k}(x)=\frac{1}{x} \int_{0}^{x} \frac{(\log x-\log u)^{\alpha-1}}{\Gamma(\alpha)} \varphi_{i}(u) d u \rightarrow 0
$$


werden, d.h. die Funktion $x^{q} e^{-x}$ ist $H^{k}$-limitierbar mit der Summe 0 . Dies gilt ebenso gut für die Funktion $\Phi(x)=\sum_{q=0}^{p-1} \frac{a_{q} x^{q} e^{-x}}{q}$. Also hat die in $\$ 1$ aufgestellte Funktion $S(x)=s(x)-\Phi(x)$ dieselbe Eigenschaft in Bezug auf $H^{k}$-Summierbarkeit wie $s(x)$, und $S^{(i)}(0)=0 \quad(i=0,1,2, \ldots$, $p-1)$ sind gültig.

\section{$\S 3$.}

Lemma 3. Es sei $k=-p+\alpha(<0)$ und $l \lessgtr 0$, dann gelten $\left({ }^{6}\right)$

$$
H^{l} H^{k} s(x)=H^{k+l} s(x) \text {, sowie } H^{k} H^{l} s(x)=H^{k+l} s(x) .
$$

$l^{\circ}$. Für positives $l$ gilt erstens

$$
\begin{aligned}
H^{l} H^{k} s(x) & =\frac{1}{x} \int_{0}^{x} \frac{(\log x-\log u)^{l-1}}{\Gamma(l)} \frac{d u}{u} \int_{0}^{u} \frac{(\log u-\log v)^{\alpha-1}}{\Gamma(\alpha)} s_{p}(v) d v \\
& =\frac{1}{x} \int_{0}^{x} \frac{s_{p}(v) d v}{\Gamma(l) \Gamma(\alpha)} \int_{0}^{x}\left(\log \frac{x}{u}\right)^{l-1}\left(\log \frac{u}{v}\right)^{\alpha-1} \frac{d u}{u} \\
& =\frac{1}{x} \int_{0}^{x} \frac{(\log x-\log v)^{l+\alpha-1}}{\Gamma(l+\alpha)} s_{p}(v) d v,
\end{aligned}
$$

womit, falls $0<l+\alpha \leqq 1$ ist, die erste Hälfte schon bewiesen ist. Es sei sonst $l+\alpha=q+\beta>1$, wo $0<\beta \leqq 1$ und $q$ positiv ganz ist. Unter Beachtung, $\operatorname{da} B \quad x s_{p}(x)=(x D)^{p}(x s(x))=x D\left(x s_{p-1}(x)\right)$ ist, erhält man

$$
\begin{gathered}
\int_{0}^{x} \frac{(\log x-\log v)^{q+\beta-1}}{\Gamma(q+\beta)} s_{p}(v) d v=\frac{1}{\Gamma(q+\beta)}\left[v s_{p-1}(v)\left(\log \frac{x}{v}\right)^{q+\beta-1}\right]_{0}^{x} \\
+\int_{0}^{x} \frac{(\log x-\log v)^{q+\beta-2}}{\Gamma(q+\beta-1)} s_{p-1}(v) d v .
\end{gathered}
$$

Der integrierte Teil verschwindet, und in gleicher Weise ergibt sich

und

$$
H^{b} H^{k} s(x)=\frac{1}{x} \int_{0}^{x} \frac{(\log x-\log v)^{\beta-1}}{\Gamma(\beta)} s_{p-q}(v) d v \quad \text { für } p \geqq q,
$$

$$
=\frac{1}{x} \int_{0}^{x} \frac{(\log x-\log v)^{q+\beta-p-1}}{\Gamma(q+\beta-p)} s(v) d v \quad \text { für } p<q,
$$

was in jedem Falle $H^{T+k} s(x)$ bedeutet.

Zweitens, falls $k=-p$ negativ ganz ist, sei bemerkt, da $B$

$$
H^{-p} \mathcal{S}(x)=\sum_{n=0}^{p} b_{n}^{(p)} x^{n} s^{(n)}(x)=s_{p}(x)=\frac{1}{x} \vartheta^{p}\{x s(x)\}, \quad\left(\vartheta \equiv x \frac{d}{d x}\right)
$$

( $\left.{ }^{6}\right)$ Für $k>0, l>0$ ist dies schon von Jacob, loc. cit., bewiesen worden. 
wird. Deshalb wird

$$
H^{-p} H^{l} s(x)=H^{-p} S(x)=S_{p}(x)=\frac{1}{x}\left\{x \frac{d}{d x}\right\}^{p}\{x S(x)\}=\frac{1}{x} \vartheta_{x}^{p}\{x S(x)\},
$$

wobei

$$
S(x)=H^{l} \delta(x)=\frac{1}{x} \int_{0}^{x} \frac{(\log x-\log u)^{l-1}}{\Gamma(l)} s(u) d u, \quad(l>0)
$$

ist, was durch $p$-malige teilweise Integration verwandelt werden kann in :

$$
S(x)=\frac{1}{x} \int_{0}^{x} \frac{(\log x-\log u)^{l+p-1}}{\Gamma(l+p)} \vartheta_{u}^{p}\{u s(u)\} \frac{d u}{u}\left(\vartheta_{u}=u \frac{d}{d u}\right) .
$$

Daraus folgt offenbar

$$
\begin{aligned}
& H^{-p} H^{l} s(x)=\frac{1}{x}\left\{x \frac{d}{d x}\right\}^{p}\{x S(x)\} \\
& \quad=\frac{1}{x} \int_{0}^{x} \frac{(\log x-\log u)^{l-1}}{I^{l}(l)} \vartheta_{u}^{p}\{u s(u)\} \frac{d u}{u}=H^{l} H^{-p} s(x)=H^{l-p} s(x) .
\end{aligned}
$$

SchlieBlich, falls $k=-p+\alpha, p$ positiv ganz, $0<\alpha<1$ und $l>0$ ist, so wird auf Grund des vorher Erwähnten

$$
\begin{aligned}
H^{l} H^{l} s(x) & =H^{\alpha-p} H^{l} s(x)=H^{\alpha} H^{-p} H^{l} s(x) \\
& =H^{\alpha} H^{l-p} s(x)=H^{a-p+l} s(x)=H^{k+l} s(x) .
\end{aligned}
$$

$\mathscr{2}^{\circ}$. Wenn $k=-p, l=-q$ beide negativ ganz sind, so ergeben sich

$$
\begin{aligned}
H^{-q} H^{-p} s(x) & =\frac{1}{x} \vartheta^{q}\left\{x H^{-p}(s) x\right\}=\frac{1}{x} \vartheta^{q}\left\{\vartheta^{p}(x s(x))\right\} \\
& =\frac{1}{x} \vartheta^{q+q}\{x s(x)\}=H^{-(p+q)} s(x), \\
H^{-r} H^{-q} H^{-p} s(x) & =H^{-r} H^{-(p+q)} s(x)=H^{-(p+q+r)} s(x),\left(r^{\prime} \text { ganz }\right), \cdots,
\end{aligned}
$$

und speziell ist

$$
H^{-1} H^{-1} \cdots(p-\text { mal }) \cdots H^{-1} s(x)=H^{-p} s(x) 。
$$

Ferner sei $k=-p+\alpha, 0<\alpha \leqq 1$; aus

$$
\begin{aligned}
H^{k} s(x) & =\frac{1}{x} \int_{0}^{x} \frac{(\log x-\log u)^{\alpha-1}}{\Gamma(\alpha)} \vartheta^{p}(u s(u)) \frac{d u}{u} \\
& =\frac{1}{x} \int_{0}^{x} \frac{(\log x-\log u)^{\alpha}}{\Gamma(\alpha+1)} \vartheta^{p+1}\{u s(u)\} \frac{d u}{u} \equiv S(x) .
\end{aligned}
$$

folgt sofort 


$$
\begin{aligned}
H^{-1} H^{k} s(x) & =S(x)+x S^{\prime}(x) \\
= & \frac{1}{x} \int_{0}^{x} \frac{(\log x-\log u)^{\alpha-1}}{\Gamma(\alpha)} \vartheta^{p+1}\{u s(u)\} \frac{d u}{u}=H^{-(p+1)+\alpha} s(x)=H^{k-1} s(x),
\end{aligned}
$$

und durch wiederholte Anwendung dieser Beziehung erhält man

$$
H^{-q} H^{k} s(x)=H^{-1} H^{-1} \cdots H^{-1} H^{k} s(x)=H^{-q+k} s(x) .
$$

Schließlich, falls $k=-p+\alpha, l=-q+\beta$ ist, wird nach dem Vorhergehenden :

$$
\begin{aligned}
H^{l} H^{k} s(x) & =H^{-q+\beta} H^{k} s(x)=H^{\beta} H^{-q} H^{k} s(x) \\
& =H^{\beta} H^{k-q} s(x)=H^{\beta+k-q} s(x)=H^{k+l} s(x), \quad \text { w.z.b.w. }
\end{aligned}
$$

Nach dem obigen Lemma verhält sich die Ordnung wie ein Exponent, was durch die Schreibweise $H^{k} s(x)$ dargestellt ist.

Lemma 4. (Konsisienzsatz). Existiert der Grenzwert $\lim _{x \rightarrow \infty} H^{k}(x)=g$, so ist auch $\lim _{x \rightarrow \infty} H^{r+k}(x)=g$, sobald $r>0$ ist.

Dies folgt unmittelbar aus dem Lemma 3. Speziell aus $H^{k}(x) \rightarrow g$, folgt $H^{k+(-k)}(x)=H^{0}(x)=s(x) \rightarrow g$, falls $k<0$ ist.

Lemma 5. Wenn die $H^{k}$-Summe der Funktion $s(x)$ existiert, wo $k=-p+\alpha, 0<\alpha \leqq 1$ ist, so verschwinden die $H^{a}$ - (und folglich $C_{\alpha^{-}}$) und auch $H^{1}$-Summen der Funktionen:

$$
x^{i} s^{(i)}(x) \quad(i=1,2, \cdots, p) .
$$

Nun folgt aus Lemma 3 und 4

$$
\begin{aligned}
H^{i} H^{k}(x) & =H^{-p+\alpha+i}(x)=H^{-(p-i)+\alpha}(x) \\
& =\frac{1}{x} \int_{0}^{x} \frac{(\log x-\log u)^{\alpha-1}}{\Gamma(\alpha)} s_{p-i}(u) d u \rightarrow g,
\end{aligned}
$$

wo $i=p, p-1, \cdots, 2,1$ ist; d.h.

$$
\begin{aligned}
& \frac{1}{x} \int_{0}^{x} \frac{(\log x-\log u)^{\alpha-1}}{\Gamma(\alpha)} s(u) d u \rightarrow g, \\
& \frac{1}{x} \int_{0}^{x} \frac{(\log x-\log u)^{\alpha-1}}{\Gamma(\alpha)}\left(s(u)+u s^{\prime}(u)\right) d u \rightarrow g, \\
& \frac{1}{x} \int_{0}^{x} \frac{(\log x-\log u)^{\alpha-1}}{\Gamma(\alpha)}\left[s(u)+3 u s^{\prime}(u)+u^{2} s^{\prime \prime}(u)\right] d u \rightarrow g, \cdots .
\end{aligned}
$$

Subtrahiert man diese nacheinander, so ergeben sich

$$
\frac{1}{x} \int_{0}^{x} \frac{(\log x-\log u)^{\alpha-1}}{\Gamma(\alpha)} u^{i} s^{(i)}(u) d u \rightarrow 0 \quad(i=1,2, \cdots, p),
$$

was eben zu beweisen war. 
Lemma 6. Wenn $H^{k}(x) \rightarrow g$ für $x \rightarrow \infty$ strebt, wobei $k=-p+\alpha, p$ positiv ganz und $0<\alpha \leqq 1$ ist, so gelten

$$
\bar{C}_{-p+i}(x) \equiv x^{p-i} s^{(p-i)}(x) \rightarrow 0 \quad(i=1,2, \cdots, p-1),
$$

und daher sind $s^{(p-i)}(x)$ alle beschränkt.

Durch teilweise Integration erhält man

$$
\begin{aligned}
\frac{1}{x} \int_{0}^{x} u^{p} s^{(p)}(u) d u=\frac{1}{x}\left[u^{p} s^{(p-1)}(u)\right. & \left.-p u^{(p-1} s^{(p-2)}(u)+\cdots+(-1)^{p-1} \mid \underline{p} u s(u)\right]_{0}^{x} \\
& +\frac{(-1)^{p} \mid \underline{p}}{x} \int_{0}^{x} s(u) d u
\end{aligned}
$$

was aber nach t.emma 5 gegen 0 streben soll, d.h.

$$
x^{p-1} s^{(p-1)}(x)-p x^{p-2} s^{(p-2)}(x)+\cdots+(-1)^{p-1}\left|\underline{p} s(x)+(+1)^{p}\right| p g \rightarrow 0 .
$$

Ebenso auch $x^{p-2} s^{(p-2)}(x)-(p-1) x^{p-s_{s}^{(p-3)}}(x)+\cdots+(-1)^{p-1} \underline{p-1} g \rightarrow 0$.

Multipliziert man den letzten Ausdruck mit $p$ und addiert ihn zum vorstehenden, so ergibt sich

$$
x^{p-1} s^{(p-1)}(x) \rightarrow 0 \text { 。 }
$$

In ganz ähnlicher Weise erhält man

$$
x^{p p-2} s^{(p-2)}(x) \rightarrow 0, \quad, x s^{\prime}(x) \rightarrow 0 .
$$

Es folgt also, für $k=-p+1 \quad(p \geqq 2)$, schon aus $H^{-p+1}(x) \rightarrow g$, dass $\bar{C}_{-p+1+1}(x) \rightarrow \frac{g}{\Gamma(2-p)}=0$. (Knoppscher Äquivalenzsatz bei negativer ganzzahliger Ordnung).

Lemma \%. Wenn $H^{k}(x) \rightarrow g$ für $x \rightarrow \infty$ strebt, wobei $k=-p+\alpha, p$ positiv gan: und $0<a<1$ ist, so gilt

(I) $\lim _{x \rightarrow \infty} x^{p-\alpha-i} \int_{0}^{x} \frac{(x-u)^{\alpha-1}}{\Gamma(\alpha)} u^{i} s^{(p)}(u) d u=\frac{(-1)^{i} \Gamma(p)}{\Gamma(p-i) \Gamma(\alpha-p+i+1)} g$,

wobei $i$ positiv ganz oder 0 und $0 \leqq i<p$ ist $\left({ }^{7}\right)$.

Für $i=p$ stimmt (I) mit Lemma 5 überein, sodass (I) durch vollständige Induktion bewiesen werden darf. Um es für $i=p-1$ zu bekommen, geht man zunächst von der Differenz aus:

$$
\begin{aligned}
x^{1-\alpha} \int_{0}^{x} \frac{(x-\cdots)^{\alpha-1}}{\Gamma(\alpha)} u^{p-1} s^{(p)}(u) d u & -x^{-\alpha} \int_{0}^{x} \frac{(x-u)^{\alpha-1}}{\Gamma(\alpha)} u s^{p(p)}(u) d u \\
& =x^{-\alpha} \int_{0}^{x} \frac{(x-u)^{\alpha}}{\Gamma(\alpha)} u^{p-1} s^{(p)}(u) d u
\end{aligned}
$$

( 7 ) Es gilt sogar $(I)$ auch für jedes ganze $i \geqq p(>0)$. Ferner gibt es eine Formel für negatives ganzes $p$. Vgl. dazu Lemma 10. 
was, teilweise integriert, liefert

$$
\begin{aligned}
x^{-\alpha} \int_{0}^{x} \frac{\alpha(x-u)^{\alpha-1}}{\Gamma(\alpha)}\left\{u_{j}^{j-1} s^{(p-1)}(u)\right. & -(p-1) u_{i}^{p-2} s^{(p-2)}(u) \\
& \left.+\cdots+(-1)^{p-1} p-1 s(u)\right\} d u
\end{aligned}
$$

da der integrierte Teil verschwindet wegen $s(0)=0$. Nun streben nach Lemma 6 und 4

$$
x^{j} s^{(j)}(x) \rightarrow 0 \quad(j=1,2, \cdots, p-1) \text { und } s(x) \rightarrow g
$$

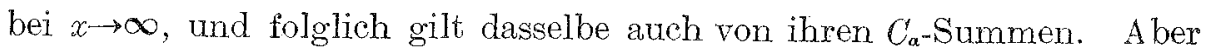
das Subtrahendenintegral strebt auch gegen 0 nach Lemma 5. Daher ergibt sich

$$
x^{1-\alpha} \int_{0}^{x} \frac{(x-u)^{\alpha-1}}{\Gamma(\alpha)} u^{p-1} s^{(p)}(u) d u \rightarrow 0(1)+\frac{(-1)^{p-1} \mid p-1}{\Gamma(\alpha)} g ;
$$

es ist also (I) gezeigt für $i=p-1$. Insbesondere bei $p=1$ ist (I) schon vollständig bewiesen, weil dann der zulässige Wert von $i$ in der genannten Grenze nur Null sein soll.

Es sei im allgemeinen angenommen, dass (I) gültig ist bej jeder Ordnung $k=\alpha-p+j(j=1,2, \cdots, p-1)$ für alle zulässigen Werte von $i$ in der genannten Grenze, d. h. für $0 \leqq i \leqq p-j-1$, und ausserdem bei der Ordnung $k=\alpha-p$ für ein gewisses $i(\leqq p-1)$. Dabei folgen nach Lemma 4 alle Teilvoraussetzungen, dass $H^{a-p+j}(x) \rightarrow g(j=1,2, \cdots, p-1)$, aus der ursprünglichen blossen Voraussetzung, dass $H^{\alpha-p}(x) \rightarrow g$. Wir kommen zum Schlusse, dass es auch für $i-1$ bei der Ordnung $k=\alpha-p$ gilt, wobei $0 \leqq i-1 \leqq p-2$, d. h. $1 \leqq i \leqq p-1$ und $p \geqq 2$ sein soll. Wir bilden dafür wiederum die Differenz:

$$
\begin{aligned}
x^{p-\alpha-i+1} \int_{0}^{x} \frac{(x-u)^{\alpha-1}}{\Gamma(\alpha)} u u^{i-1} s^{(p)}(u) d u & -x^{p-\alpha-i} \int_{0}^{x} \frac{(x-u)^{\alpha-1}}{\Gamma(\alpha)} u^{i} s^{(p)}(u) d u \\
& =x^{p-\alpha-i} \int_{0}^{x} \frac{(x-u)^{\alpha}}{\Gamma(\alpha)} u^{i-1} s^{(p)}(u) d u
\end{aligned}
$$

was, teilweise integriert, und unter Berücksichtigung, dass $s^{(J)}(0)=0(j=0$, $1,2, \cdots, p-1$ ) sind, wird

$$
\begin{aligned}
& x^{p-\alpha-i} \int_{0}^{x} \frac{\alpha(x-u)^{\alpha-1}}{\Gamma(\alpha)} \sum_{j=0}^{i-1} \frac{(-1)^{j} \Gamma(i)}{\Gamma(i-j)} u^{i-j-1} s^{(p-j-1)}(u) d u \\
& =\alpha \sum_{j=0}^{i-1} \frac{(-1)^{j} \Gamma(i)}{\Gamma(i-j)} x^{(p-j-1)-\alpha-(i-j-1)} \int_{0}^{x} \frac{(x-u)^{\alpha-1}}{\Gamma(\alpha)} u^{i-j-1} s^{(p-j-1)}(u) d u
\end{aligned}
$$


wobei $0 \leqq i-j-1 \leqq p-j-2<p-j-1$ wegen $i \leqq p-1$, und folglich $p-1 \geqq$ $p-j-1 \geqq 1$ wegen $p \geqq 2$ ist. In dieser Grenze ist (I) gültig nach den jetzigen Annahmen. Somit strebt der obige Summenausdruck gegen

$$
\alpha \sum_{j=0}^{i-1} \frac{(-1)^{j} \Gamma(i)}{\Gamma(i-j)} \cdot \frac{(-1)^{i-j-1} \Gamma(p-j-1)}{\Gamma(p-i) \Gamma(\alpha-p+i+1)} g .
$$

Hiermit bleibt nur zu zeigen, dass der letztere Summenausdruck gleich der Differenz

$$
\frac{(-1)^{i-1} \Gamma(p)}{\Gamma(p-i+1) \Gamma(\alpha-p+i)} g-\frac{(-1)^{i} \Gamma(p)}{\Gamma(p-i) \Gamma(\alpha-p+i+1)} g
$$

ist, denn das Subtrahendenintegral strebt schon gegen den letzteren Subtrahenden nach unserer Annahme. D. h. es genügt zu beweisen, dass

oder dass

$$
\begin{aligned}
\alpha \sum_{j=0}^{i-1} \frac{\mid i-1}{\underline{i-j-1}} \cdot \frac{\mid p-j-2}{\underline{p-i-1} \Gamma(\alpha-p+i+1)} & =\frac{\mid p-1}{\underline{\underline{p-i} \Gamma(\alpha-p+i)}} \\
& +\frac{\mid p-1}{\mid \underline{p-i-1} \Gamma(\alpha-p+i+1)}
\end{aligned}
$$

$$
\sum_{j=0}^{i-1} \frac{\mid p-j-2}{\mid i-j-1}=\frac{\mid p-1}{(p-i) \mid i-1}
$$

ist, was offenbar gilt, falls $i=p-1$; andernfalls, wenn $i-j-1=m$ und $p-i-1=n$ gesetzt werden, wobei $p \geqq 3$ sein soll, weil $p-1>i \geqq 1$ ist, verwandelt sich der Ausdrucke in

$$
\frac{1}{n} \sum_{m=0}^{p-n-2} \frac{\mid m+n}{\underline{m}}=\frac{(p-1)(p-2) \cdots(p-n-1)}{\mid n+1}
$$

was aber eine bekannte Identität ist. Damit ist alles bewiesen.

Der Knoppsche Äquivalenzsatz bei negativer gebrochener Ordnung:

Setzt man $i=0$ in $(\mathrm{I})$ des vorigen Lemmas, so folgt

$$
\lim _{x \rightarrow \infty} x^{p-\alpha} \int_{0}^{r} \frac{(x-u)^{\alpha-1}}{\Gamma(\alpha)} s^{(p)}(u) d u=\frac{g}{\Gamma(\alpha-p+1)} ;
$$

also wenn bei $x \rightarrow \infty, H^{k}(x) \rightarrow g$ ist, so folgt stets $\bar{C}_{k}(x) \rightarrow \frac{g}{\Gamma(k+1)}$,w.z.b.w. (Bei negativer ganzer Ordnung ist es schon in Lemma 6 bewiesen).

\section{§4。}

Um die andere Hälfte des Äquivalenzsatzes (Schneescher Äquivalenzsatz) zu beweisen, wollen wir zuerst bekommen, was aus $\bar{C}_{k}(x) \rightarrow \frac{g}{\Gamma(k+1)}$ 
folgt. Um jedoch brauchbare Ergebnisse zu erhalten, scheint es notwendig, falls $k \leqq-1$ ist, einige Zusatzbedingungen anzunehmen. In der Tat ist es klar, dass aus $\bar{C}_{-p+1}(x) \rightarrow 0$ nicht $H^{-p+1}(x) \rightarrow g$ folgt, da $g$ in der Voraussetzung nicht vorkommt. Aber auch für nichtganzes $k<-1$ braucht aus $C_{k}(x) \rightarrow g$ nicht $H^{k}(x) \rightarrow g$ zu folgen. Das Hausdorffsche Beispiel $\left({ }^{8}\right)$ für solche Zahlenfolgen karn auch auf jenes für Funktionen ausgedehnt werden. Ist nämlich $s(x)=\int_{0}^{x} \frac{(x-u)^{-k-1}}{\Gamma(-k)}(u+1)^{t} d u$ $=\sigma^{-k}(x+1)^{k}$, wo $k=-p+\alpha, p \geqq 2,0<\alpha<1$ ist, so ist nach Lemma 1

$$
C_{k}(x)=\frac{\Gamma(k+1) \sigma^{k}(x)}{x^{k}}=\frac{\Gamma(k+1)}{2^{k}} \sigma^{k} \sigma^{-k}(x+1)^{k}=\Gamma(k+1)\left(\frac{x+1}{x}\right)^{k} \rightarrow \Gamma(k+1) .
$$

Jedoch hat man

$$
\begin{aligned}
s(x)>\int_{0}^{1} \frac{(x-u)^{p-\alpha-1}}{\Gamma(p-\alpha)}(u+1)^{\alpha-p} d u>2^{\alpha-p} \int_{0}^{1} \frac{(x-u)^{p-\alpha-1}}{\Gamma(p-\alpha)} d u \\
=\frac{2^{\alpha-p}}{\Gamma(p-\alpha+1)}\left[x^{p-\alpha}-(x-1)^{p-\alpha}\right] \rightarrow+\infty
\end{aligned}
$$

weil $p-\alpha>1$ ist, also nicht einmal $s(x) \rightarrow \Gamma(k+1)$, geschweige denn $H^{k}(x) \rightarrow \Gamma(k+1)$.

Lemma 8. Wenn $\bar{C}_{k}(x) \rightarrow \frac{q}{\Gamma(k+1)}$ ist, wo $k=-p+\alpha, p$ positiv ganz $\geqq 2$ und $0<\alpha \leqq 1$ ist, mit den. Zusätzbedingungen, dass alle $s^{(i)}(x)(i=0$, $1,2, \cdots, p-2)$ beschränkt sind, so folgt

$$
\bar{C}_{k+j}(x) \rightarrow \frac{g}{\Gamma(k+j+1)} \quad(j=1,2, \cdots, p-2) .
$$

Falls $0<\alpha<1$ ist $\left({ }^{9}\right)$, so gilt es auch für $j=p-1$ und es folgt sogar $s(x) \rightarrow g$.

Fall $1^{\circ}: 0<\alpha<1, p \geqq 2$. Nach Lemma 1

$$
\sigma^{k+1}(x)=\sigma \sigma^{k}(x)=\int_{0}^{x} \sigma^{k}(x) d x
$$

Es ist aber

$$
\sigma^{k}(x)=O\left(x^{k}\right)
$$

da $\bar{C}_{k}(x)$ existiert. Überdies ist $k<-1$. Daher existiert auch:

$$
\lim _{x \rightarrow \infty} \sigma^{x+1}(x)=\int_{0}^{\infty} \sigma^{x}(x) d x=l
$$

( $\left.{ }^{8}\right)$ Hausdorff loc. cit., siehe S. 191.

(9) Wenn $k>-1$, also $p=1,0<\alpha<1$ ist, so ist die Sache schon bei Lemma 2 betrachtet worden. Ebenso für $j>\mathrm{p}-1$. 
Um jedoch die Existenz der $\left.\bar{C}_{k+1}{ }^{\prime} x\right)$ festzustellen, soll etwas anderes angenommen werden. Während Hausdorff das Abelsche Verfahren annimmt, wåhle ich folgende Transformation für das Integral:

$$
T f=\lim _{t \rightarrow+0} t \int_{a}^{+\infty} e^{-u t} f(u) d u .
$$

Es ist $T f \rightarrow l$, falls $f(u) \rightarrow l$ für $u \rightarrow \infty$ strebt. Denn, wird $f(u)=l+\delta(u)$ gesetzt, so wird $|\delta(u)|<\varepsilon$ für $u \geqq v$, und

$$
\begin{aligned}
t \int_{0}^{\infty} e^{-u t} f(u) d u & =\int_{0}^{p}+\int_{\nu}^{\infty}=0(t)+t \int_{\nu}^{\infty} e^{-3 t t}(l+\delta(u)) d u \\
& =0(1)+l e^{-y t}+0\left(t \int_{\nu}^{\infty} e^{-u t} d t\right) \rightarrow l
\end{aligned}
$$

bei $t \rightarrow 0$. Wenn $f(u)$ limitierbar ist, so darf dieser Limes nichts anderes als $T f$ sein. Nun betrachtet man $T \sigma^{k+1}(x)$ :

$$
\begin{aligned}
t \int_{0}^{\infty} e^{-x t} \sigma^{k+1}(x) d x & =t \int_{0}^{\infty} e^{-x t} d x \int_{0}^{x} \frac{(x-u)^{\alpha-1}}{\Gamma(\alpha)} s^{(p-1)}(u) d u \\
& =t \int_{0}^{\infty} s^{(p-1)}(u) d u \int_{u}^{\infty} \frac{(x-u)^{\alpha-1}}{\Gamma(\alpha)} e^{-x t} d x
\end{aligned}
$$

was durch Einsetzung $(x-u) t=v$ wird

$$
\begin{aligned}
& t \int_{0}^{\infty} \frac{s^{(p-1)}(u) d u}{\Gamma(\alpha)} \int_{0}^{\infty} t^{-\alpha} v^{\alpha-1} e^{-u t-v)} d v=t^{1-\alpha} \int_{0}^{\infty} e^{-u t s^{(p-1)}}(u) d \tau_{b} \\
& \quad=t^{1-\alpha}\left[e^{-u t}\left(s^{(p-2)}(u)+t s^{(p-3)}(u)+\cdots+t^{p-2} s(u)\right)\right]_{0}^{\infty}+t^{p-\alpha} \int_{0}^{\infty} e^{-u t} s(u) d u .
\end{aligned}
$$

Wegen der Zusatzbedingungen, dass $\delta^{(i)}(u)$ beschränkt sind, strebt der integrierte Teil gegen 0 . Ebenso verschwindet das letztere Integral für $t \rightarrow 0$. Daraus folgt

$$
\lim _{x \rightarrow \infty} \sigma^{x+1}(x)=l=0, \quad \sigma^{k+1}(x)=-\int_{x}^{\infty} \sigma^{k}(u) d u
$$

Da aber nach Voraussetzung

ist, so exgibt sich

$$
\sigma^{k}(x) \cong \frac{g x^{k}}{\Gamma(k+1)}
$$

$$
\sigma^{\bar{k}+1}(x)=-\int_{x}^{\infty}\left[\frac{g}{\Gamma(k+1)}+\delta(u)\right] u^{k} d u=\frac{g x^{k+1}}{\Gamma(k+2)}+0\left(\int_{x}^{\infty} u^{k} d u\right) \quad(k<-1)
$$

und daher wird

$$
\vec{C}_{k+1}(x) \rightarrow \frac{q}{\Gamma(k+2)}
$$


Ebenso folgen $\quad \bar{C}_{k+i}(x) \rightarrow \frac{q}{\Gamma(k+j+1)} \quad(j=1,2, \cdots, p-1)$.

Speziell für $j=p-1$ gilt

$$
\bar{C}_{a-1}(x) \rightarrow \frac{g}{\Gamma(\alpha)},
$$

wo $0>\alpha-1>-1$ ist, und so folgt nach Lemma 2

$$
\bar{C}_{0}(x)=s(x) \rightarrow g \text {. }
$$

Fall $\otimes^{\circ}: \quad \alpha=1, k=-p+1, p>2$. In diesem Falle wird die Voraussetzung

$$
\bar{C}_{k}(x)=x^{p-1} s^{(p-1)}(x) \rightarrow 0(1)
$$

In ganz gleicher Weise wie in Fall $1^{\circ}$, kann es gezeigt werden, dass

$$
\bar{C}_{k+j}(x)=x^{p-j \sim 1} s^{(p-j-1)}(x) \rightarrow 0 \quad(j=1,2, \cdots, p-2)
$$

sind. (Für den Fall $p=2, \alpha=1$. gibt es kein solches $j$, ausser $j=0$.)

Bemerkung. Falls $\alpha=1$ ist, so folgt aus $\bar{C}_{-p+1}(x) \rightarrow 0$, keineswegs, dass $\bar{C}_{0}(x)=s(x) \rightarrow g$ ist. Andererseits folgt in diesem. Falle jedoch, dass

für $x \rightarrow \infty$ ist. Denn es. ist

$$
\frac{1}{x} \int_{0}^{x} u^{p} \delta^{(p)}(u) d u \rightarrow 0(1)
$$

$$
\begin{aligned}
\frac{1}{x} \int_{0}^{x} u^{p-1} s^{(p-1)}(x) d u & =\frac{1}{p x}\left[u^{p} s^{(p-1)}(u)\right]_{0}^{x}-\frac{1}{p x} \int_{0}^{x} u^{n} s^{(p)}(u) d u \\
& =\frac{1}{p} x^{p-1} s^{(p-1)}(x)-\frac{1}{p x} \int_{0}^{x} u^{p} s^{(p)}(u) d u,
\end{aligned}
$$

was im ganzen gegen 0 streben soll, da $x^{p^{p-1} s^{(p-1)}}(x) \rightarrow 0$ ist.

Lemma 9. Vorausgesetzt, dass $C_{k}(x) \rightarrow g$ für $x \rightarrow \infty$ strebt, wobei $k=$ $-p+\alpha, p$ positiv ganz und $0<\alpha<1$ ist, und ausserdem, dass, falls $p \geqq 2$, auch $s^{(l)}(x)(l=0,1,2, \cdots, p-2)$ alle beschränkt sind, so folgt wieder die Beziehung ausgedrückt in Lemma $r$ :

(I) $\lim _{x \rightarrow \infty} x^{p-\alpha-i} \int_{0}^{x} \frac{(x-u)^{\alpha-1}}{\Gamma(\alpha)} u^{i} s^{(p)}(u) d u=\frac{(-1)^{i} \Gamma(p) g}{\Gamma(p-i) \Gamma(\alpha-p+i+1)}$,

wobei $i$ positiv ganz oder 0 und $0 \leqq i \leqq p-1$ ist.

Für $i=0$ stimmt (I) mit der Voraussetzung überein. Daher gilt (I), für $p=1$ schon vollständig in der genannten Grenze von $i$, da alsdann $\dot{i}=0$ der einzige zulässige Wert sein soll.

Es sei im allgemeinen angenommen, dass (I) gültig ist bei jeder Ordnung $k=-p+\alpha+j(j=1,2, \cdots, p-1)$ für alle zulässigen Werte von $i$ in der genannten Grenze, d. h. für $0 \leqq i \leqq p-j-1$, und ausserdem bei 
der Ordnung $k=-p+\alpha$ für ein geweisses $i-1$, wobei $0 \leqq i-1 \leqq p-2$, d.h. $1 \leqq i \leqq p-1$ und $p \geqq 2$ sein soll. Dabei folgen nach Lemma 8 alle Teilvoraussetzungen bei $p \geqq 2$, dass $C_{-p+a+j}(x) \rightarrow g(j=1,2, \cdots, p-1)$, aus der ursprïnglichen Voraussetzung $C_{-\uparrow+a}(x) \rightarrow g$ mit Zusatzbedingungen, dass $s^{(l)}(2)(l=0,1,2, \cdots, \mu,-2)$ beschränkt sind: $\operatorname{lm}$ von $i-1$ auf $i$ zu schliessen, verfährt man ganz ähnlich wie in Lє mma 7 , lediglich mit dem trivialen Unterschiede, dass der Limes des Subtrahenden anstatt des Limes des Minuenden gesucht ist.

Lemma 10. Aus der Voraussetzung in Lemma 9 (oder Lemma ${ }^{7}$ ) folgt ferner

$$
\lim _{x \rightarrow \infty} x^{p-\alpha-i} \int_{0}^{x} \frac{(x-u)^{\alpha-1}}{\Gamma(\alpha)} u^{i} s^{(x)}(u) d u=0
$$

falls $i$ ganz und $i \geqq p(\geqq 1)$ ist. Übrigens gilt

$$
\text { (III) } \lim _{x \rightarrow \infty} x^{-q-\alpha-i} \int_{0}^{x} \frac{(x-u)^{a-1}}{\Gamma(\alpha)} u^{i} s^{(-\alpha)}(u) d u=\frac{\Gamma(q+i+1)}{\Gamma(q+1) \Gamma(q+\alpha+i+1)} g,
$$

wobei $q$ und $i$ irgendwelche positive ganze Zahlen oder 0 bedeuten und

$$
s^{(-\alpha)}(x)=\int_{0}^{x} d u_{1} \int_{0}^{u_{1}} d u_{2} \cdots \int_{0}^{u q^{-1}} s(u) d u=\int_{0}^{x} \frac{(x-u)^{q-1}}{\Gamma(q)} s(u) d u \quad\left(s^{(0)}(x)=s(x)\right)
$$

ist.

Im wesentlichen ist (II) in (I) enthalten, weil der Faktor $T(p-i)$ in dem Nenner auf der rechten Seite von (I) unendlich gross wird, falls $p-i$ eine negative ganze Zahl oder 0 ist. Nur der Bequemlichkeit wegen hatte ich sie getrennt.

Die Beziehung (III) spielt die Rolle eines Hilfsmittel zum Beweise von (II)... Sie folgt unmittelbar aus der blossen Annahme, dass $s(x)$ bei $x \rightarrow \infty$ gegen $g$ strebt, was wahr ist nach Lemma 8 (oder nach Lemma 4. falls wir das Lemma 7 ausdehnen wollen), wenn $C_{-p+\alpha}(x)$ (oder $H^{-p+a}$ (x)) bei $x \rightarrow \infty$ vorhanden ist. In der Tat ist $s(x)$ alsdann $C_{q}$-summierbar mit der Summe $g$, d. $h$.

wåhrend

$$
\lim _{x \rightarrow \infty} \mid q x^{-q} s^{(-q)}(x)=g
$$

$$
\lim _{x \rightarrow 0} \frac{s^{(-q)}(x)}{x^{q}}=\lim _{x \rightarrow 0} \frac{s(x)}{\underline{q}}=0 ;
$$

also die Funktion $x^{-q} s^{(-q)}(x)$ ist durchaus stetig in dem ganzen Intervall $(0,+\infty)$. Nach einem bekannten Satz von Chapman $\left({ }^{10}\right)$ haben wir deshalb für irgendeine Zahl $r(>-1)$

(10) Hardy-Riesz, loc. cit. p. 27, oder Chapman, loc. cit. 


$$
\lim _{x \rightarrow \infty} \frac{\Gamma(r+\alpha+1)}{\ldots x^{r+a}} \int_{0}^{x} \frac{(x-u)^{a-1}}{\Gamma(\alpha)} \cdot \frac{u^{r-q} s^{(-\alpha)}(u)}{\Gamma(r+1)} d u=\frac{g}{q}
$$

oder, falls $r-q=i(>-(q+1))$ gesetzt wird, so gilt

$$
\lim _{x \rightarrow \infty} x^{-q-i-\alpha} \int_{0}^{x} \frac{(x-u)^{\alpha-1}}{\Gamma(\alpha)} u^{i} s^{(-\alpha)}(u) d u=\frac{\Gamma(q+i+1) g}{\Gamma(q+1) \dot{\Gamma}(q+i+\alpha+1)},
$$

was, für positives ganzes oder versehwindendes $i$, eben (III) gibt.

Dies sei vorausgeschickt, und nun wollen wir (II) durch Schluss von $i-1$ auf $i$ beweisen, wobei $i \geqq p \geqq 1$ ist. Dafür bilden wir nochmals die Differenz:

$$
\begin{array}{r}
x^{p-\alpha-i+1} \int_{0}^{x} \frac{(x-u)^{\alpha-1}}{\Gamma(\alpha)} u^{i-1} y^{(p)}(u) d u-x^{p-\alpha-i} \int_{0}^{x} \frac{(x-u)^{\alpha-1}}{\Gamma(\alpha)} u^{i} s^{(p)}(u) d u \\
=x^{p-\alpha-i} \int_{0}^{x} \frac{(x-u)^{\alpha}}{\Gamma(\alpha)} u^{i-1} s^{(p)}(u) d u \\
=\alpha \sum_{j=0}^{i-1} \frac{(-1)^{j} \Gamma(i)}{\Gamma(i-j)} x^{p-\alpha-i} \int_{0}^{x} \frac{(x-u)^{\alpha-1}}{\Gamma(\alpha)} u^{i-j-1} s^{(1)-j-1)}(u) d u \equiv \sum .
\end{array}
$$

In dem Falle $p=1, i \geqq 1$ ergibt sich nach (III)

$$
\begin{aligned}
\lim _{x \rightarrow \infty} \sum & =\alpha \sum_{j=0}^{i-1} \frac{(-1)^{j} \Gamma(i)}{\Gamma(i-j)} \cdot \frac{\Gamma(i)}{\Gamma(j+1) \Gamma(\alpha+i)} g \\
& = \begin{cases}g / \Gamma(\alpha) & \text { für } i=1, \\
\frac{\alpha g \Gamma(i)}{\Gamma(\alpha+i)}(1-1)^{i-1}=0 & \text { für } i>1 .\end{cases}
\end{aligned}
$$

Das Mạnuendenintegral auf der äussersten linkẹn Sẹite für $p=i=1$ strebt gegen $g / \Gamma(\alpha)$ nach $(\mathrm{I})$, also. wird

$$
x^{-\alpha} \int_{0}^{x} \frac{(x-u)^{\alpha-1}}{\Gamma(\alpha)} u s^{\prime}(u) d u i \rightarrow 0
$$

bei $x \rightarrow \infty$, d. h. (II) gilt für $p=1, i=1$. Durch Einsetzung $p=1, i=2$ in die obige Differenz, erkennen wir dann, dass: (II) auch für $p=1, i=2$, u. s. w. gilt. Also besteht (II) für jedes ganzzahlige $i \geqq p=1$.

In dem Falle $i \geqq p \geqq 2$, zerlegen wir die obigé Summation

wobei

$$
\Sigma=\Sigma^{\prime}+\Sigma^{\prime \prime}
$$

$$
\begin{aligned}
& \Sigma^{\prime}=\alpha \sum_{j=0}^{n-2} \frac{(-1)^{j} \Gamma(i)}{I(i-j)} x^{p-j-1-(i-j-1)-\alpha} \int_{0}^{x} \frac{(x-u)^{\alpha-1}}{\Gamma(\alpha)} u^{i-j-1} s^{(p-j-1)}(u)^{i} d u \\
& \Sigma^{\prime \prime}=\alpha \sum_{j=p-1}^{i-1} \frac{(-1)^{j} T(i)}{\Gamma(i-j)} x^{-(j-p+1)-(i-j-1)-\alpha} \int_{0}^{x} \frac{(x-u)^{\alpha-1}}{I(\alpha)} u^{i-j-1} s^{-(j-j+1)}(u) d u
\end{aligned}
$$


ist. Erstens, in $\sum^{\prime}$ ist $i-j-1 \geqq p-j-1>0$ und $p-j-1 \leqq p-1$. Angenommen, dass (II) für alle derartigen $i \geqq p-j-1 \geqq 1:(j=0,1,2, \cdots$, $p-2)$ gültig ist, so strebt jedes Glied in $\Sigma^{\prime}$ gegen 0 und deshalb.

$$
\sum \rightarrow 0
$$

bei $x \rightarrow \infty$. Zweitens, in $\sum^{\prime \prime}$ ist $i-j-1 \geqq 0$ and $j-p+1 \geqq 0$, sodass nach (III) wird

$$
\begin{aligned}
\Sigma^{\prime \prime} & =\alpha \sum_{j=p-1}^{i-1} \frac{(-1)^{j} \Gamma(i)}{\Gamma(i-j)} \cdot \frac{\Gamma(i-p+1) g}{\Gamma(j-p+2) \Gamma(\alpha-p+i+1)} \\
& =\frac{\alpha g \Gamma(i)}{\Gamma(\alpha-p+i+1)} \sum_{j=p-1}^{i-1} \frac{(-1)^{j} \Gamma(i-p+1)}{\Gamma(j-p+2) \Gamma(i-j)} \\
& = \begin{cases}\frac{\alpha g(-1)^{n-1} \Gamma(p)}{\Gamma(\alpha+1)}=\frac{(-1)^{p-1} I(p)}{\Gamma(\alpha)} g & \text { für } \quad i=p, \\
\frac{\alpha g(-1)^{p-1} \Gamma(i)}{\Gamma(\alpha-p+i+1)}(1-1)^{i-p}=0 & \text { für } i>p .\end{cases}
\end{aligned}
$$

Für $i=p$, nach dem Resultat, welches man durch Einsetzung des Wertes $i=p-1$ in (1) exhält, strebt also das Minuendenintegral auf dex äussersten linken Seite gegen den hintersten Ausdruck auf der rechten Seite. Somit ergibt sich

$$
\lim _{x \rightarrow \infty} \frac{1}{x^{a}} \int_{0}^{x} \frac{(x-u)^{a-1}}{\Gamma(\alpha)} u^{n} s^{(x)}(u) d u=0
$$

d. h. die Funktion $x^{p_{3}(p)}(x)$ ist $C_{a}$-summierbar mit der Summe $0\left({ }^{11}\right)$.

In dem Falle $i>p$, unter der weiteren Annahme, dass (II) gültig ist bei gegebenem $p$ für gewisses $i-1$ (z. B. $i-1=p)$, verschwindet das Minuendenintegral auf der äussersten linken Seite und daraus folgt

$$
\lim _{x \rightarrow \infty} x^{p-\alpha-i} \int_{0}^{x} \frac{(x-u)^{\alpha-1}}{\Gamma(\alpha)} u^{i} s^{(p)}(u) d u=0 ;
$$

also (II) ist gültig bei jenem $p$ auch für $\dot{t}$. Daher gilt im allgemeinen (II) bei jedem ganzen Werte von $p$ für jedes ganze $i \geqq p$.

Der Schneesche Aquivalenzsatz bei negativer Ordnung:

Um dies $z u$ beweisen, betrachten wir die folgenden Fälle getrennt:

a) Fall $0>k=\alpha-1>-1$. Aus $C_{l}(x) \rightarrow g$ ohne Zusatzbedingung folgt $C_{-k+k}(x)=s(x) \rightarrow g$ nach Lemma 2, und daher auch $H^{\alpha}(x) \rightarrow g$. Die

(11) Unter Zusammenfassung von Lemma 9 und 10 folgt dies aus $C \rightarrow p+\alpha(x) \rightarrow g$, mit den Zusatzbedingungen bei $p \geqq 2$, dass $\varepsilon^{(l)}(x)(l=0,1,2, \cdots, p-2)$ beschränkt sind Jedoch fïr $p=1$ bedarf es keiner Zusatzbedingung. 
Funktion $x s^{\prime}(x)$ ist nach dem obigen Lemma $C_{\alpha}$-limitierbar und folglich auch $I I^{\alpha}$-limitierbar( ${ }^{1 *}$ ) mit der Summe 0. Daraus folgt, dass

$$
H^{k}(x)=\frac{1}{x} \int_{0}^{x} \frac{(\log x-\log u)^{\alpha-1}}{\Gamma(\alpha)}\left\{s(u)+u \delta^{\prime}(u)\right\} d u \rightarrow g .
$$

b) Fall $k=-p+\alpha<-1,0<\alpha<1, p \geqq 2$. Unter Voraussetzung

$$
C_{k}(x)=\frac{\Gamma(k+1)}{x^{k}} \int_{0}^{x} \frac{(x-u)^{\alpha-1}}{\Gamma(\alpha)} s^{(p)}(u) d u \rightarrow g
$$

mit den Zusatzbedingúngen, dass $s^{(v)}(x)(l=0,1,2, \cdots, p-2)$ beschränkt sind, soll bewiesen werden, dass bej $x \rightarrow \infty$

strebt, wobei

$$
I^{k}(x)=\frac{1}{x} \int_{0}^{x} \frac{(\log x-\log u)^{\alpha-1}}{\Gamma(\alpha)} s_{p}(u) d u \rightarrow y
$$

$$
s_{p}(x)=\sum_{n=0}^{p} b_{n}^{(p)} x^{n} s^{(n)}(x) \quad\left(b_{0}^{(p)}=1\right)
$$

ist. Es ist nun nach Lemma 8

$$
C_{-p+a+j}(x) \rightarrow g \quad(j=0,1,2, \cdots, p-1) .
$$

Somit ist nach Lemma 10 jede Funktion $2^{p-j^{(p-j)}}(x)(j=0,1,2, \cdots, p-1)$ $C_{a}$ - und auch $H^{a}$-summierbar mit der Summe 0, während die Funktion $s(x)$, sowie ihre $H^{a}{ }^{a}$ Summe gegen $g$ strebt. Daher ergibt sich

$$
H^{k}(x)=\frac{1}{x} \int_{0}^{x} \frac{(\log x-\log u)^{a-1}}{\Gamma(\alpha)} s_{p}(u) d u \rightarrow g .
$$

c) Fall $k=-p+1 \leqq-1$. In diesem Fall ist die Voraussetzung

$$
\bar{C}_{k}(x)=x^{p-1} s^{(p-1)}(x) \rightarrow 0,
$$

sogar mit den Zusatzbedingungen, dass $s^{(l)}(x)(l=0,1,2, \cdots, p-2)$ beschränkt sind, noch nicht genug um zu erlangen, dass $H^{k}(x) \rightarrow g$ ist, obwohl zwar $x^{j} s^{(j)}(x) \rightarrow 0(j=1,2, \cdots, p-1)$ folgen. Aber, mit der weiteren Bedingung, dass $s(x) \rightarrow g$ ist, erhält man

$$
\begin{aligned}
H^{k}(x) & =H^{-p+1}(x)=\frac{1}{x} \int_{0}^{x} s_{p}(u) d u \\
=\frac{1}{x} \int_{0}^{x} s(u) d u+\sum_{j=1}^{p-1} b_{j}^{(p)} \frac{1}{x} \int_{0}^{x} u^{j} s^{(j)}(u) d u & +\frac{1}{x} \int_{0}^{x} u^{p} s^{(p)}(u) d u \\
& =g+0(1)+0(1) .
\end{aligned}
$$

(12) Jacob, loc. cit. 
Auf das letztere Glied ist nun die Bemerkung am Ende von Lemma 8 anzuwenden.

Zusatz. Es sei $C_{k}(x) \rightarrow g$, wo $k=-p+\alpha, 0<\alpha<1, p$ positiv ganz $\geqq 2$ und $s^{(l)}(x)(l=0,1,2, \cdots, p-2)$ beschränkt sind. Dann gilt $\bar{C}_{k+\varepsilon}(x) \rightarrow$ $\frac{g}{\Gamma(k+\varepsilon+1)}$, sobald $\varepsilon>0$ ist (Konsistenzsatz). Wenn $p \geqq 2, \alpha=1$ ist, so gilt dasselbe mit der ferneren Nebenbedingung, dass $s(x) \rightarrow g$. (Für den Fall $p=1$ ist es schon in Lemma 2 bewiesen).

Denn, nach letzten Hälfte des Aquivalenzsatzes, ist $H^{*}(x) \rightarrow g$. Folglich ist auch $H^{k+\varepsilon}(x) \rightarrow g$ nach Lemma, 4, woraus $\bar{C}_{k+\varepsilon}(x) \rightarrow \frac{g}{\Gamma(k+\varepsilon+1)}$

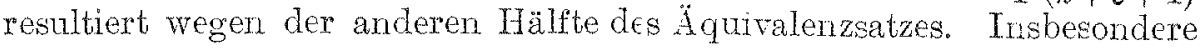
bei $p \geqq 2$ für $\varepsilon=-k-j=p-\alpha-j(j=1,2, \cdots, p-1)$ ergeben sich

$$
\bar{C}_{x+\varepsilon}(x)=\bar{C}_{-j}(x)=x^{j} s^{(j)}(x) \rightarrow \frac{g}{\Gamma(1-j)}=0 \quad(j=1,2, \cdots, p-1),
$$

was, für den Fall $\alpha=1$, schon in Lemma 8 erhalten worden sind.

Zum Schlusse sei es mir gestattet Herrn M. Tzuji meinen besten Dank auszusprechen für seine freundlichen Ratschläge, die es mir ermöglichten einige Unstimmigkeiten der ursprünglichen Fassung und schwachen Punkte des Beweisgangs zu beseitigen.

Yokohama Techn. College. 